\title{
Research Progress in Determination Methods of Formaldehyde Content in Indoor Air
}

\author{
Bowei Yao, Hairui Wang, Wei Zhang, Lili Feng \\ Ningxia Academy of Metrology and Quality Inspection, Ningxia, Yinchuan, 750001 \\ DOI: $10.32629 /$ aes.v2i4.543
}

\begin{abstract}
The detection methods of formaldehyde content in indoor air, including traditional laboratory detection methods (AHMT spectrophotometry, phenol reagent spectrophotometry, acetyl acetone spectrophotometry and gas chromatography) and rapid detection methods (electrochemical sensor method, photoelectric spectrophotometry, etc.), were introduced and described. This paper systematically analyzes and compares the detection principle, applicable environment medium, detection flux and the advantages and disadvantages of each detection method. The future detection methods of formaldehyde content in indoor air were prospected.
\end{abstract}

Keywords: indoor air, formaldehyde, detection method

\section{Introduction}

Formaldehyde (HCHO) is a common volatile organic pollutant in the air, known as the number one "killer"of interior decoration ${ }^{[1]}$. Literature data show that long-term exposure to low concentration of formaldehyde will lead to a series of diseases such as chromosomal abnormalities in newborns, memory decline in adolescents, chronic respiratory diseases, leukemia, and nasopharyngeal cancer. Formaldehyde has been identified as a carcinogen and has genetic toxicity ${ }^{[2]}$. Therefore, the detection of formaldehyde content in indoor air is more and more concerned by people, and its significance is more and more important. In this paper, the conventional and rapid detection methods of formaldehyde content in indoor air reported at home and abroad are summarized as follows.

\section{The harm and source of formaldehyde}

\subsection{Harm of formaldehyde}

Formaldehyde, also known as ant aldehyde, is a colorless gas with strong pungent smell at room temperature ${ }^{[3]}$. It is easily soluble in water, and its aqueous solution, formalin, is often used as a preservative $\mathrm{e}^{[4]}$. Formaldehyde is highly toxic and causes great harm to human experience, including symptoms of olfactory disorders, skin irritation and decreased immune function ${ }^{[5]}$. In the list of carcinogens published by the World Health Organization agency for Cancer Research in October 2017, formaldehyde was listed in the list of class I carcinogens ${ }^{[6]}$.

\subsection{Sources of formaldehyde}

As the main raw material of the adhesive, formaldehyde is widely used in various home decoration materials to play a bonding role. Studies have shown that ${ }^{[7]}$ all kinds of artificial boards such as particleboard, density board and wallpaper are the main sources of formaldehyde in indoor air.

\section{Determination method for formaldehyde content in indoor air}

Indoor Air Quality Standard GB/T 18883-2002 ${ }^{[8]}$ stipulates that the concentration limit of formaldehyde in indoor air is $0.1 \mathrm{mg} / \mathrm{m}^{3}$ and Indoor Environmental Pollution Control Standard for Civil Construction Engineering GB 50325-2020 ${ }^{[9]}$ stipulates that the formaldehyde content of class I civil construction engineering should be $\leq 0.07 \mathrm{mg} / \mathrm{m}^{3}$. Class ii civil construction engineering formaldehyde content should be less than or equal to $0.08 \mathrm{mg} / \mathrm{m}^{3}$. Therefore, it is necessary to use efficient and accurate detection methods to detect the formaldehyde content in indoor air, so as to evaluate the indoor air quality, and take relevant measures to control and reduce the harm of formaldehyde to human body.

\subsection{Regular detection methods for formaldehyde content in laboratory}

\subsubsection{AHMT spectrophotometry}

AHMT, 4- amino-3 - hydrazine -5- hydrophobic -1, 2, 4- triazobene. Formaldehyde in air was condensed with AHMT ( i ) under alkaline conditions ( ii ) and oxidized by potassium periodate to form 6-phobyl-5 triazobene [4, 3-B] -S-tetraa- 
zobenzene (iii) purply-red compound. At $550 \mathrm{~nm}$, the color depth of the compound was proportional to the formaldehyde content, so as to determine the formaldehyde content ${ }^{[10]}$. The method has the advantages of good specificity and selectivity. It has no influence on the method when a large number of aldehydes such as acetaldehyde, propyl aldehyde and benzaldehyde coexist with methanol and ethanol, and the detection limit is $0.04 \mathrm{mg} / \mathrm{L}$. The disadvantage is that the color solution gradually deepens over time in the process of operation, and the color reaction time of the standard solution and the sample solution must be strictly unified, resulting in poor reproducibility and difficulty in operation ${ }^{[11]}$.

\subsubsection{Phenol reagent spectrophotometry}

Phenol reagent method ${ }^{[12]}$, the principle of which is that formaldehyde reacts with phenol reagent in air to generate azine, which is oxidized by high iron ion in acidic solution to form blue-green compounds, and colorimetric quantification is carried out according to the color depth. The advantages of this method are high sensitivity and the detection limit is 0.015 $\mathrm{mg} / \mathrm{L}$. The disadvantage is that the phenol reagent is not stable, it needs to be reconfigured after being stable in the refrigerator for 3 days, and the determination process is susceptible to the interference of sulfur dioxide in the air.

\subsubsection{Acetyl acetone spectrophotometry}

The main principle of acetyl acetone method ${ }^{[13]}$ is that formaldehyde gas is absorbed by water and reacts with acetyl acetone in acetic acid-ammonium acetate buffer solution of $\mathrm{pH}=6$. Under the condition of boiling water bath, stable yellow compounds are rapidly generated. The absorbance value is measured at $413 \mathrm{~nm}$ and compared with the standard series for quantification. The equation is shown in Figure 1. The advantages of this method are not disturbed by acetaldehyde, stable performance, convenient and simple operation process. But it also has some disadvantages, the color time needs about 60 min, the detection speed is slow.<smiles>CC(=O)CC1=CNC=C(CC(=O)[CH]C=O)C1</smiles>

Figure 1. Reaction principle of acetylacetone method

The above three methods are formaldehyde reacts with different detection reagents to form different color complexes. Spectrophotometer is used for colorimetric quantification at specific absorption wavelengths, and formaldehyde content is calculated according to sample absorbance and standard curve. In general, spectrophotometric detection is of low sensitivity, complex operation, low detection flux and susceptible to interference of other substances.

\subsubsection{Gas chromatography}

The principle of gas chromatography ${ }^{[12]}$ to detect formaldehyde in air is that formaldehyde is adsorbed by 2, 4-dinitrophenylhydrazine (2, 4-DNPH) 6201 carrier under acidic conditions to generate a stable formaldehyde-hydrazone. After elution with carbon disulfide, it was separated by OV- column and determined by hydrogen flame ionization detector. The retention time was qualitative and peak height was quantitative. The method has high sensitivity and the lowest detected mass concentration is $0.01 \mathrm{mg} / \mathrm{m}^{3}$.

\subsubsection{High performance liquid chromatography}

High performance liquid chromatography ${ }^{[14]}$ was used to determine formaldehyde. The principle was to use a sample tube filled with 2, 4-DNPH to collect a certain volume of air samples. Aldehydes and ketones in the samples reacted with DNPH coated on silica gel catalyzed by strong acid to generate stable colored hydrazone derivatives. The retention time was qualitative and the peak area was quantitative by HPLC with UV $(360 \mathrm{~nm})$ or diode array detector. The equation is shown in Figure 2. The blank standard addition recovery of this method is $98.6 \%-101 \%$, and the accuracy is high.

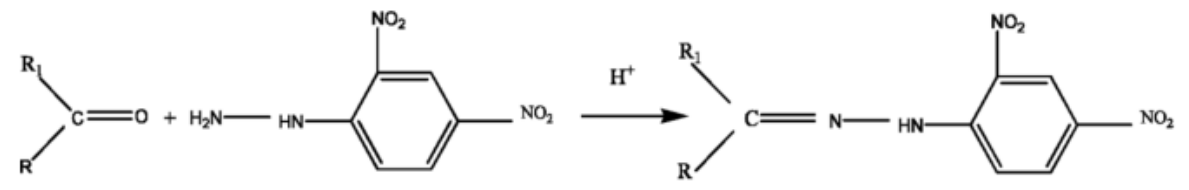

Figure 2. Reaction principle of HPLC method 
Compared with spectrophotometry, the above two chromatographic methods have low detection limit and high sensitivity, but rely on large precision instruments, expensive cost, higher requirements for experimental personnel, low detection flux, and can not achieve real-time monitoring.

\subsection{Rapid detection method for formaldehyde content}

Spectrophotometric method and chromatography is the widely used laboratory formaldehyde detection method, the common disadvantage is that the samples are required to pass through the early stage of the site, again by the laboratory for sample treatment and instrumental analysis, process trival, operation time is long, the cost of testing instruments and levels of personnel the demand is higher, low detection flux, unable to realize real-time dynamic monitoring, which fails to meet the requirements of mass testing.

With the rapid improvement of urbanization speed and the popular awareness of environmental protection, the public demand for formaldehyde detection in indoor air is increasing day by day. Traditional laboratory analysis and detection methods can no longer meet the real-time field detection demand. Rapid formaldehyde content detection method represented by portable formaldehyde detector is favored by researchers and inspectors for its advantages of fast detection speed and large flux ${ }^{[15]}$, and has been more and more applied to formaldehyde content detection in indoor air.

\subsubsection{Electrochemical sensor method}

The principle of electrochemical sensor method ${ }^{[12]}$ is that formaldehyde gas passes through the sensor, and under the action of electrolyte catalysis, formaldehyde molecules undergo REDOX reaction on the electrode to form electron transfer, and a current proportional to formaldehyde concentration is formed under the action of external voltage, so as to determine formaldehyde content, and the value of formaldehyde content can be obtained on the sampling site. The advantages of this method are that it can realize real-time field detection and detection speed is fast and efficiency is high. However, it has high environmental requirements. It can be used under $25 \%-75 \%$ humidity and is affected by coexistence gases such as $\mathrm{H}_{2} \mathrm{~S}, \mathrm{SO}_{2}$, ammonia and methanol.

\subsubsection{Photoelectric photometric method}

The principle of photoelectric photometry ${ }^{[12]}$ is that when formaldehyde gas passes through the detection unit, the color of the test paper in the detection unit changes from white to yellow due to chemical reaction, and the change of reflected light intensity caused by the degree of discoloration is in a functional relationship with formaldehyde content, and the value of formaldehyde content can be obtained on the sampling site. The advantages of this method are real-time detection, fast detection speed, high efficiency; However, its accuracy is low, the total uncertainty is less than $25 \%$ compared with phenol reagent method, and it is affected by $\mathrm{CO}, \mathrm{CO}_{2}$, acetaldehyde, acetone and other coexistence gases.

\subsubsection{Other rapid formaldehyde detection methods and their applications}

Lu Qingluan et al. ${ }^{[16]}$ compared formaldehyde rapid detector with acetyl acetone spectrophotometry to detect formaldehyde, and found that there was no significant difference in the detection results of the two methods, with $\mathrm{p}$ values greater than 0.5 . However, the formaldehyde detector is greatly affected by the environment, and the detection results are not accurate on foggy days and rainy days, and it is easy to receive the influence of interfering substances.

Li Gang et al. ${ }^{[17]}$ used formaldehyde rapid detector and acetyl acetone spectrophotometry to detect formaldehyde, and found that under 95\% confidence, SPSS paired T test showed no significant difference between the two detection data. However, it has certain influence on the measured values under high humidity. The measured values are high in the range of relative humidity $>90 \%$, and the average relative error is $41.8 \%$. To ensure the accuracy of test data, avoid using the rapid detector in foggy days, rainy days and other high-humidity environments.

Xinghua Chang et al. ${ }^{[18]}$ developed a formaldehyde detector based on zno-3\% $\mathrm{SnSO}_{4}$ nanosensor, which can detect ultra-low concentration of formaldehyde $(0.06 \mathrm{ppm})$ with good selectivity and can detect formaldehyde in indoor air.

Xin Yang et al. ${ }^{[19]}$ developed a formaldehyde detection system for indoor air based on G-quadruplex DNAzyme and Smart phone. The system could detect FA as low as $0.01 \mu \mathrm{M}$ with a linear range of $1-600 \mu \mathrm{M}$. Taking advantages of smartphone and DNAzyme, the assay provides great potential for its practical application as a home testing or on-site analysis with high sensitivity and selectivity.

Yasuko Yamada Maruo et al. ${ }^{[20]}$ developed a yellow product of the reaction between 1-phenyl-1, 3-butandione and catalyzed oxide portable formaldehyde detection device. The device can realize every half hour or one hour real-time monitoring of formaldehyde content in indoor air. The detection limit was $5 \mu \mathrm{gm}-3 \mathrm{~h}$.

At the same time, the literature data ${ }^{[21-22]}$ shows that the sample inspection of several portable formaldehyde detectors on the market shows that the value error and detection repeatability in the setting environment do not meet the standard requirements. 
Portable formaldehyde detector, in general, is convenient to carry, with easy operation is and fast detection and big flux, which can realize the real-time dynamic monitoring on the spot. However, there are some requirements for the environmental humidity. There are various brands in the market with different performances and quality. There is no unified operational procedures, which needs to be further standardized and improved on the formaldehyde detector equipment standards.

\section{Conclusion}

At present, the detection methods of formaldehyde content in indoor air can be roughly divided into spectrophotometry, chromatography and portable detector method. Spectrophotometry and chromatography enjoys high accuracy, good sensitivity and suitable for various environments. However, the cost of the equipment and the requirements for the detection personnel are high, and the detection cycle is long, so the real-time monitoring cannot be realized. Portable detector method has the advantages of convenient operation, low cost, short detection cycle and so on. It has great advantages in realizing real-time field monitoring. Moreover, it can be combined with intelligent equipment to provide better testing experience for the public and form a beneficial supplement to traditional laboratory testing methods.

\section{Acknowledgments}

This article was supported by the project of Ningxia Academy of Metrology and Quality Inspection: "High-throughput Microplate Method and Technology Research for the Detection of Formaldehyde Content in Indoor Air". The project No. is $\mathrm{KY}(2019) 06 \mathrm{Y}$.

\section{References}

[1] Zhang Jun. Detection and control of indoor formaldehyde pollution[J]. Journal of Petroleum Educational Institute of Xinjiang, 2004(01):98-99.

[2] Li Tianbao, Ji Fang, Wang Yingzi. Research progress of harm and detection methods of aldehydes[J]. Leather and Chemicals, 2017, 34(05):24-27.

[3] Li Jianrong, Zhu Junli. Research progress of endogenous formaldehyde in food[J]. Journal of Chinese Institute of Food Science and Technology, 2011, 11(09):247-257.DOI:10.16429/j.1009-7848.2011.09.017.

[4] Wolkoff P.Indoor air pollutants in office environments: Assessment of comfort, health, and performance[J]. International Journal of Hygiene \& Environmental Health, 2013, 216 (4) : 371-394.

[5] Ning Xiaoyu, Yuan Xianghua, Zheng Hao, Lu Zhiqiang.Research progress in the treatment of formaldehyde pollution[J]. China Environmental Protection Industry, 2010(05):23-25.

[6] Ying L, Yan Y, Lei X Y, et al. Purification and characterisation of two enzymes related to endogenous formaldehyde in Lentinula edodes[J]. Food Chemistry, 2013, 138(4):2174-2179.

[7] Zhang Maoliang, Wang Jinhua, Wan Xindi, Yang Yanjuan, Zhang Liping, Li Shuling. Adsorption effect of different bentonite on formaldehyde in air[J]. New Building Material, 2017, 44(07):27-29+36.

[8] GB/T 18883-2002, Indoor Air Quality Standard[S].

[9] Indoor Environmental Pollution Control Standard for Civil Building Engineering GB 50325-2020[S].

[10] Standard method for hygienic examination of formaldehyde GB/T 16129-1995 in the atmosphere of residential area spectrophotometric method[S].

[11] Shang Xiuli, Feng Wencheng, Suo Longning, Chen Shufen, Tang Rongping. Detection and prevention of formaldehyde pollutants in interior Decoration materials[J]. China Building Materials Science and Technology, 2013(05):12-16.

[12] Public Health Inspection Method GB/T 18204.2-2014, -- Part 2: Chemical pollutants[S].

[13] GB/T 15516-1995, Air quality determination of formaldehyde acetyl acetone spectrophotometric method[S].

[14] HJ/T 683-2014, Determination of aldehydes and ketones in ambient air by high performance liquid chromatography[S].

[15] Ding Songyan. Related research progress of indoor formaldehyde pollution inspection technology[J]. Chemical Industry Times, 2017, 31(11): 37-39+ 48.

[16] Lu Qingluan, Lu Xifeng, Man Jie, Wang Miaomiao. Comparative study of indoor formaldehyde rapid detector method and acetyl acetone spectrophotometry[J]. Chemical Industry Times, 2018, 32(09):20-23.

[17] Li Gang, Li Juan, Wen Xia, Zheng Mengyue. Comparison of indoor formaldehyde rapid detector method and acetyl acetone spectrophotometric method[J]. Refrigeration and Air-Conditioning (Sichuan), 2015, 29(05):523-527.

[18] Xinghua Chang, Xiuqi Wu, Yanru Guo, et al. $\mathrm{SnSO}_{4}$ modified $\mathrm{ZnO}$ nanostructure for highly sensitive and selective formaldehyde detection[J]. Sensors and Actuators B: Chemical, 2018, 255:1153-1159.

[19] Yang X, Wang Y, Liu W, et al. A portable system for on-site quantification of formaldehyde in air based on G-quadruplex halves coupled with A smartphone reader[J]. Biosensors and Bioelectronics, 2016. 
[20] Yasuko Yamada Maruo, Jiro Nakamura. Portable formaldehyde monitoring device using porous glass sensor and its applications in indoor air quality studies[J]. Analytica Chimica Acta, 2011, 702(2):247-253.

[21] No spectral in Net red formaldehyde detector[J]. Quality and Standardization, 2019(03):52.

[22] Zhang Chunyi.Formaldehyde detector no longer be in no use[N]. China Quality Daily, 2019-02-28(004). 